\title{
Bistability and discontinuity in the tunnel current of two-dimensional electron-hole layers
}

\author{
A. Parlangeli, P. C. M. Christianen, and J. C. Maan \\ Research Institute for Materials, High Field Magnet Laboratory, University of Nijmegen, Toernooiveld 1, \\ 6525 ED Nijmegen, The Netherlands \\ M. Henini \\ Department of Physics, University of Nottingham, NG7 2RD Nottingham, United Kingdom \\ (Received 11 November 1999; revised manuscript received 7 July 2000; published 26 February 2001)
}

\begin{abstract}
We present a detailed study of a recently reported discontinuity and bistability in a 12-nm GaAs/AlAs single-barrier $p-i-n$ heterostructure, where a system of spatially separated two-dimensional electron and hole $(e-h)$ layers of equal and tunable density is realized. Both features appear at $T \$ 300 \mathrm{mK}$ and are strongly enhanced in a magnetic field $B \gtrsim 10$ T perpendicular to the layers, whereas they are suppressed by $B \sim 1 \mathrm{~T}$ parallel to the layers. They correspond to a discontinuity in the $e-h$ density and in the phase of the current magneto-oscillations. Whereas the high-current state has the expected properties of the uncoupled $e-h$ layers, the low-current state behaves anomalously under all circumstances, and we identify them with a gas of spatially indirect excitons with binding energy $0.03 \mathrm{meV} \leq E_{b} \lesssim 0.3 \mathrm{meV}$ and $0.5 \mathrm{meV} \lesssim E_{b} \lesssim 5 \mathrm{meV}$ at $B$ $=0$ and $B=10 \mathrm{~T}$, respectively. We interpret the bistability as a transition between the two regimes, which arises because of the competition between the in-plane screening, determined by the average $e-e(h-h)$ distance and the magnetic length, and the interlayer $e-h$ attraction. [S0163-1829(00)08143-1]
\end{abstract}

DOI: $10.1103 /$ PhysRevB.63.115307

PACS number(s): 73.21.-b, 71.35.-y, 73.40.Gk

\section{INTRODUCTION}

In the search for Bose-Einstein condensation, spatially separated two-dimensional (2D) electron-hole $(e-h)$ layers of equal density have been intensively studied in the last few years. ${ }^{1-20}$ In these systems, the $e-h$ recombination time can be made as long as $1 \mu \mathrm{sec}$, therefore allowing thermalization of the carriers to the lattice temperature, a situation that is never reached in strictly 2D $e-h$ systems because of the higher recombination rate. With respect to real atoms, ${ }^{21}$ indirect excitons are particularly attractive because of their light mass, which is expected to allow condensation at $T$ $\sim 1 \mathrm{~K}$, rather than in the $\mu \mathrm{K}$ regime. ${ }^{5}$ In addition, the condensation is favored by a magnetic field perpendicular to the layers. ${ }^{8,9,17}$ To date, the only evidence for exciton condensation in those systems, although in the presence of a random potential, has been reported by Butov et al. ${ }^{5,6}$ In Ref. 5, a detailed discussion of the physics involved is presented.

The interest in $2 \mathrm{D} e-h$ layers is not only restricted to this controversial topic. Because of the difficulties in engineering suitable structures, most of the work done so far has been theoretical, resulting in an extremely complex scenario, which is still experimentally largely unexplored. Depending on the interlayer distance, density, applied magnetic field, and temperature, the electronic configuration is expected to range from the normal exciton gas to the excitonic superfluid, ${ }^{7-12,17}$ the dipole density wave, ${ }^{13}$ or separate fractional quantum Hall states. ${ }^{11,12}$

Recently, technology has allowed one to make semiconductor heterostructures where closely spaced 2D $e$ and $h$ layers can be actually realized. To date, most of the experimental work has been done on the optical properties of undoped double quantum wells (DQW's), ${ }^{4-6,14-16}$ which provide a natural tool for obtaining charge separation. As already realized by Kuramoto and Horie, ${ }^{17}$ another attractive possibility is to study the transport ${ }^{1,2,20}$ and optical ${ }^{3}$ properties of $p-i-n$ single-barrier heterostructures (SBH's), in which 2D $e-h$ layers of equal density accumulate under forward bias at opposite sides of the barrier.

With respect to DQW's, $p-i-n$ SBH's have several advantages. First of all, for sufficiently thick barriers $(\sim 10 \mathrm{~nm}$ for GaAs/AlAs structures), recombination and tunneling times are much longer than the carrier relaxation times, and the carriers are able to reach the lattice temperature. In other words, the system is expected to reach thermodynamic quasiequilibrium, in which the chemical potentials of the $e$ and $h$ gases are separately aligned to the ones in the respective doped regions. Additional advantages are the possibility of tuning the density with the applied voltage and the presence of only two interfaces, which allows high in-plane mobility.

We studied a 12-nm-thick single-barrier $p-i-n$ GaAs/ AlAs/GaAs heterostructure (Fig. 1) ${ }^{1-3}$ In such a device, two limits are known. At low densities $\left(n \sim 10^{10} \mathrm{~cm}^{-2}\right)$, Neves et $a l .{ }^{20}$ have observed resonant tunneling via indirect exciton

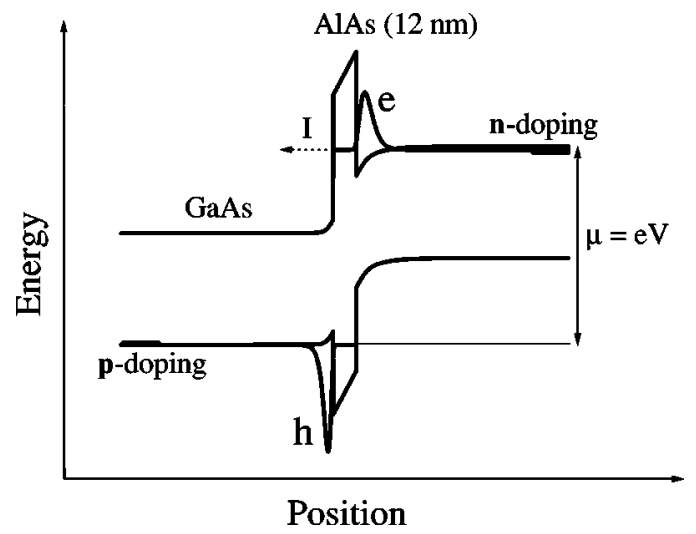

FIG. 1. Band diagram of the sample studied. The GaAs undoped layers are $300 \mathrm{~nm}$ thick and are not plotted in scale. 
states, which manifests itself as peaks in the low-temperature $(T \lesssim 10 \mathrm{~K}) I(V)$ curves. At high densities, on the other hand, Teisser and co-workers ${ }^{22,23}$ reported the behavior of uncoupled $e-h$ layers. We studied the intermediate regime $n \sim 10^{11} \mathrm{~cm}^{-2}$, i.e., when the in-plane $e-e(h-h)$ distance is comparable to the interlayer $(e-h)$ one. We observed bistability and discontinuity in the tunnel current, ${ }^{1,2}$ which we believe to arise from a phase transition between the two regimes. A synthesis of our results was reported in Refs. 1,2, and we present here a more detailed study discussing all the experimental data measured.

The paper is organized as follows: we describe the sample in Sec. II, and we show in Sec. III experimental results of the temperature dependence (Sec. III A), the magnetooscillations at constant bias (Sec. III B), the magnetic field dependence (Sec. III C), and the in-plane magnetic field (Sec. III D). In Sec. IV we discuss the experimental results and, while discussing other possibilities, we show that a suitable model to explain the data is based on the transition from a gas of indirect excitons and the uncoupled $e-h$ layers. Finally, we present our conclusions in Sec. V.

\section{SAMPLE}

The layer composition of the device we studied is $2 \mu \mathrm{m}$ p-doped GaAs $\left(4 \times 10^{18} \mathrm{~m}^{-2}\right), 100 \mathrm{~nm}$ of weaker doping $\left(1 \times 10^{17} \mathrm{~m}^{-2}\right), 100 \mathrm{~nm}$ undoped GaAs, $11.9 \mathrm{~nm}$ AlAs barrier, $100 \mathrm{~nm}$ undoped GaAs, $100 \mathrm{~nm} n$-doped GaAs (2 $\left.\times 10^{16} \mathrm{~m}^{-2}\right)$, and $500 \mathrm{~nm} n$-doped GaAs $\left(4 \times 10^{18} \mathrm{~m}^{-2}\right)$. The sample was grown by molecular beam epitaxy in the [311] crystallographic direction in order to ensure high inplane hole mobility. We measured similar samples grown on the [100] direction, but they did not show the discontinuities discussed in this paper, although some smeared feature appeared at high magnetic field also in this case. The sample was processed in circular mesas with optical access. The results discussed in this paper were measured in a $50-\mu \mathrm{m}$ mesa, but the main features were reproduced on the other ones.

A schematic band diagram of the device under forward bias is shown in Fig. 1. We stress that, in this structure, the applied voltage is proportional to the chemical potential $\mu_{e-h}=e V$ of the $e-h$ system, which is defined as the energy required to add an $e-h$ pair. We estimated the $e-h$ distance $d_{e-h} \sim 25 \mathrm{~nm}$ from the distance between the maxima of the wave functions, which we obtained by self-consistent calculations in the Fang-Howard approach.

In the regime of our interest, only the lowest heavy-hole and electron subbands are populated. ${ }^{24}$ We measured the tunnel current in the growth direction, which is determined by electrons (holes have a much smaller tunneling probability because of their bigger effective mass). The optical transition between the spatially separated $e-h$ layers (a potentially very useful spectroscopic tool) is totally negligible.

\section{EXPERIMENT}

The $I(V)$ characteristics of the device, at $B=0$ ( $B$ is the external magnetic field) and $T=4.2 \mathrm{~K}$, are shown in

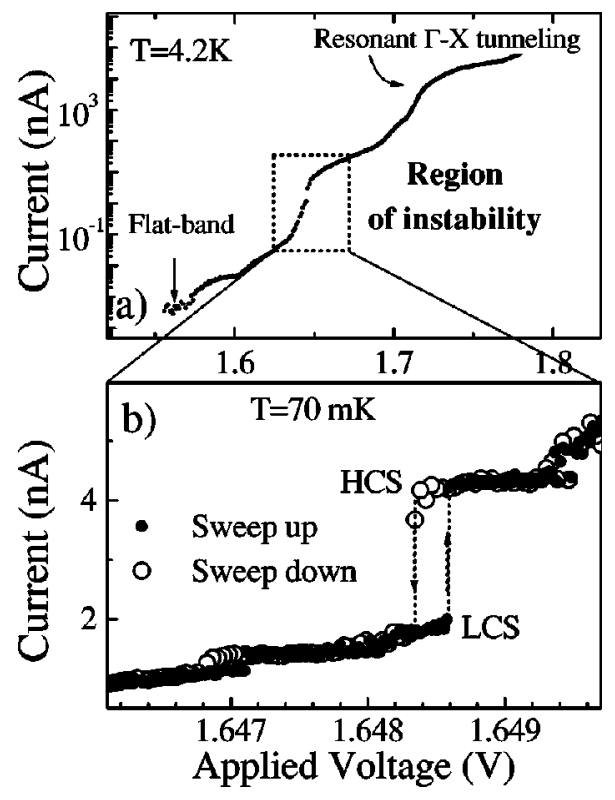

FIG. 2. (a) $I(V)$ characteristics at $T=4.2 \mathrm{~K}$ and $B=0$. The regime of high current was studied by Teisser et al. (Ref. 23), whereas the regime of low current, close to flatband conditions, was studied by Neves et al. in Ref. 20. We focused on the intermediate regime delimitated by a square (Refs. 1 and 2). (b) Bistability of the tunnel current at $B=0$ and $T=70 \mathrm{mK}$. The measured points are shown as solid and open circles for sweep up and down, respectively. The electronic states are named the "high-current state", (HCS) in the upper branch and the "low-current state" (LCS) in the lower one.

Fig. 2(a). Flat band occurs at $\sim 1.52 \mathrm{~V}$, as expected for GaAs. At slightly higher biases $(\sim 1.55$ to $\sim 1.6 \mathrm{~V})$, Neves et $a l .{ }^{20}$ observed peaks in the tunnel current, which they attributed to resonant tunneling from indirect excitons across the barrier to direct excitons in the collector. The regime of high bias has been studied by Finley et la $^{22}$ in relation to $\Gamma-X$ tunneling; we observed a very similar behavior of our structure for biases higher than $\sim 1.7 \mathrm{~V}$. In the intermediate regime, delimited by a square in Fig. 2(a), we observed a feature $^{1,2}$ that has never been observed on $n-i-n$ devices of similar layer sequence (see, for instance, Refs. 25-27). We believe therefore that such a feature, which corresponds to $d_{e-e} \sim d_{e-h} \approx 25 \mathrm{~nm},{ }^{28}$ originates from the presence of the additional hole layer.

When the temperature is further decreased to $T$ $=70 \mathrm{mK}$ and $B=0$, the feature becomes discontinuous and bistable, as shown in Fig. 2(b). ${ }^{1,2}$ We denote the states corresponding to the upper and lower branch of the bistability the "high-current state" (HCS) and "low-current state" (LCS), respectively.

\section{A. Temperature dependence and stability}

In order to gain further insight into the energy scale of the bistability, we investigated the temperature dependence of the $I(V)$ curves. The results are plotted in Fig. 3 and show that if the temperature increases, the bistability reduces in size and shifts to lower biases and higher currents (the curve corresponding to $T \sim 50 \mathrm{~K}$ is offset for comparison). 


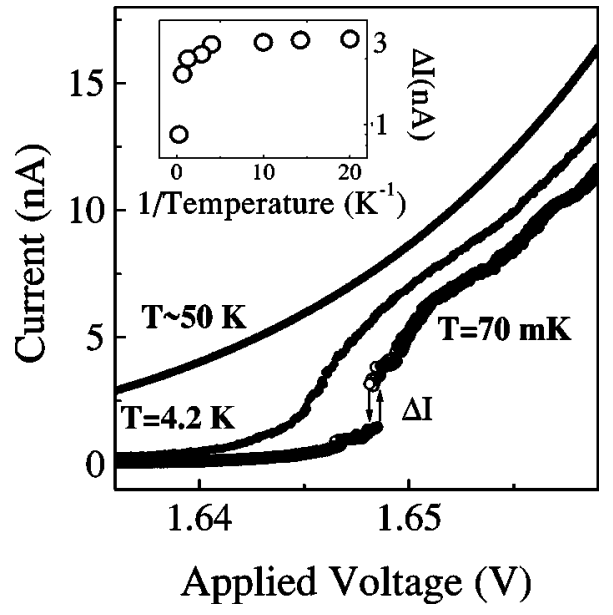

FIG. 3. $I(V)$ curves at $B=0$ and $T=70 \mathrm{mK}, 4.2 \mathrm{~K}$, and $\sim 50 \mathrm{~K}$. At $T=70 \mathrm{mK}$, the sweep up and down are indicated by solid and open circles, respectively. In the inset, we show the Arrhenius plot of the current difference $\Delta I$ between the HCS and LCS at the center of the transition.

Both the bistability and the discontinuity disappear at temperatures higher than $T^{*} \sim 300 \mathrm{mK}(\sim 30 \mu \mathrm{eV})$, but a smeared feature in the $I(V)$ survives up to $T=4.2 \mathrm{~K}$ $(\sim 0.5 \mathrm{meV})$. At temperatures around $50 \mathrm{~K}$, the single exponential behavior typical of a $p-i-n$ diode is recovered.

In the inset of Fig. 3, we show the current difference $\Delta I$ (in logarithmic scale), measured at the center of the transition, between the HCS and LCS. The voltage position and bistability width have a similar behavior, which saturates at low temperatures $T \sim 800 \mathrm{mK}$.

The saturation might be either intrinsic to the physical process involved or due to the saturation of the electronic temperature to $\sim 800 \mathrm{mK}$. We do not have the possibility of discriminating between the two effects, and throughout the whole paper, we therefore only report the bath temperature.

In the presence of a magnetic field $B=10 \mathrm{~T}$ perpendicular to the layers, the bistability width, the current discontinu-

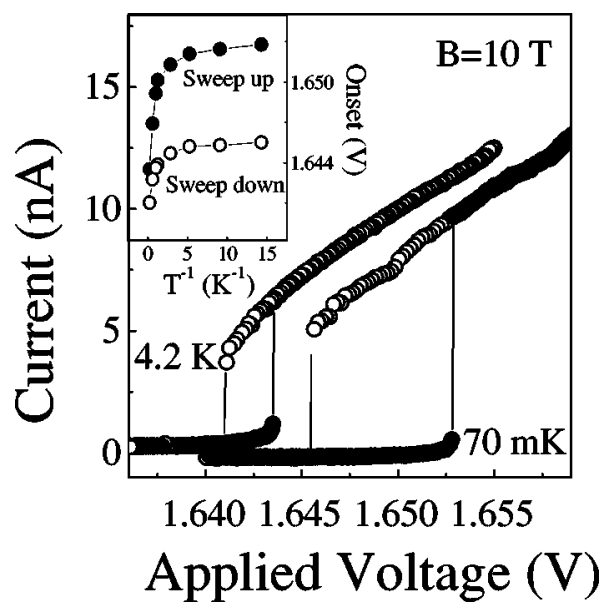

FIG. 4. $I(V)$ curves at $T=70 \mathrm{mK}$ and $4.2 \mathrm{~K}$, in a magnetic field $B=10 \mathrm{~T}$ perpendicular to the layers. The sweep up and down are indicated by solid and open circles, respectively. In the inset, we show the Arrhenius plot of the discontinuity positions.

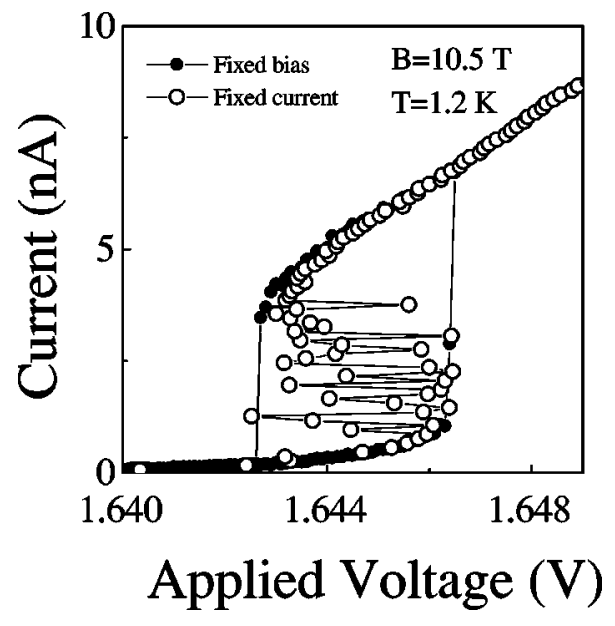

FIG. 5. Comparison between the $I(V)$ curves (solid circles) and the Voltage measurements at fixed current (open circles). The temperature is $4.2 \mathrm{~K}$ and the magnetic field is $B=10 \mathrm{~T}$ (perpendicular to the layers).

ity, and the temperature $T^{*}$ increase by an order of magnitude, as shown in Fig. 4. Also in this case, upon an increase of temperature, the bistability reduces in size and shifts to lower biases and higher currents. The temperature behavior of the voltage position (inset of Fig. 4), current discontinuity, and bistability width is similar to the one at $B=0$, but we observe bistability up to $T=4.2 \mathrm{~K}$, which points to an energy scale of the order of $1 \mathrm{meV}$, one order of magnitude bigger than at $B=0$.

At $B=10.5 \mathrm{~T}$, we have also studied the stability of the LCS and HCS. Both the states are stable at constant bias, for at least $15 \mathrm{~min}$; transitions from either of the states to the other could be triggered by electrical noise. In contrast, if we apply a fixed current in the region of negative differential conductance (1-5 nA), we observe that $V$ is unstable between the two values $(1.646 \mathrm{~V}$ and $1.653 \mathrm{~V})$ at which the discontinuity occurs (Fig. 5).

\section{B. Magneto-oscillations of the tunnel current}

We have further characterized the LCS and HCS by measuring the tunnel current as a magnetic field perpendicular to

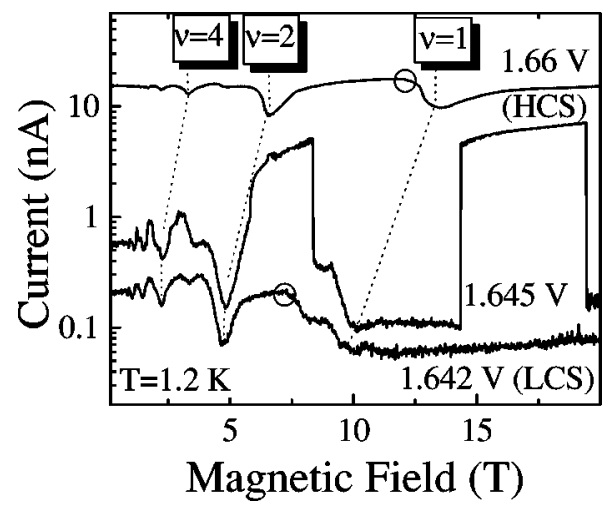

FIG. 6. Magneto-oscillation (MO) traces for $V=1.66 \mathrm{~V}$ (HCS), $V=1.642 \mathrm{~V}$ (LCS), and $V=1.645 \mathrm{~V}$ (both LCS and HCS). Maxima positions at $1<\nu<2$ are marked by open circles. 
the layers swept at fixed applied voltage (i.e., at fixed chemical potential $\left.\mu_{e-h}=e V\right) .{ }^{25}$ In Fig. 6, three typical traces measured at $T=1.2 \mathrm{~K}$ are shown: one in the LCS $(1.642 \mathrm{~V})$, another in the HCS $(1.66 \mathrm{~V})$, and an intermediate one at the transition $(1.645 \mathrm{~V})$. These sweeps show general properties and do not strictly refer to Fig. 2, since the details of the bistability (position, width, and current discontinuity) depend on temperature and magnetic field, as discussed above.

The magneto-oscillations (MO's) of the tunnel current arise from the effect of the magnetic field $B$ on the density of states (DOS) of the two-dimensional electron gas (2DEG), which becomes quantized in Landau levels (LL's) with degeneracy $e B / h$, separated by the cyclotron energy $\hbar \omega_{c}$ $=\hbar e B / m$ ( $m$ is the effective mass). The physical properties of the system are determined by the filling factor $\nu$ $=n h / e B$ ( $n$ is the density), which is the number of occupied LL's. The Fermi energy $E_{F}$ of the 2DEG is pinned to both the lowest occupied LL and to the chemical potential (fixed by the applied voltage) in the doped regions. This condition induces a self-consistent rearrangement of the band profile when $B$ is swept, ${ }^{25}$ which modulates the tunneling current with periodicity $E_{F} / \hbar \omega_{c} \propto 1 / B$. The minima occur approximately at integer filling factor (we resolve spin splitting), at fields given by

$$
B_{\nu}=\frac{n h}{e \nu}=\frac{B_{1}}{\nu} .
$$

From analysis of Eq. (1), we found that the HCS and LCS have very different behavior. The values of the densities deduced from Eq. (1) are shown in Fig. 7(a), and the behavior is clearly nonlinear at the transition. The HCS oscillations can be described by Eq. (1), with a linear dependence of $n$ on $V$, as expected from a normal 2DEG (Ref. 23) [dotted line in Fig. 7(a)]. In contrast, the LCS shows a systematic deviation, and a linear $1 / B$ dependence can only be obtained when $\nu$ is replaced by $\nu-\alpha[0.2 \leq \alpha \leq 0.25$; see the inset of Fig. 7(b) for $V=1.642 \mathrm{~V}]$, corresponding to a phase shift of $60^{\circ}-90^{\circ}\left(\alpha^{*} 360^{\circ}\right)$ [Fig. 7(b)].

Furthermore, in the bistable region, the LCS and the HCS show different densities at the same applied voltage (i.e., at the same chemical potential), as can be seen by sweeping $B$ around the $\nu=1$ minimum in both directions. In Fig. 3 of Ref. 1, we plot both curves together for $V=1.646 \mathrm{~V}$. We observe that the minimum in the MO is realized at a lower field (thus lower density) for the LCS $(10.5 \pm 0.1 \mathrm{~T})$ than for the HCS $(11.5 \pm 0.5 \mathrm{~T})$, and that the difference between the densities is about $10 \%$. The HCS density deduced from Fig. 3 of Ref. 1 is plotted in Fig. 7(a) with a square.

When the temperature is further decreased to $70 \mathrm{mK}$, small dips appear in the HCS MO at $\nu=2 / 3$, as shown in Fig. 8. These minima are not visible in the LCS (Fig. 8) and disappear at $T=1.2 \mathrm{~K}$ (Fig. 6); moreover, they allow an accurate determination of the HCS density at the transition. The values of the density obtained in this way naturally extend the ones deduced from the analysis of the full $1 / B$ periodicity at $T=1.2 \mathrm{~K},{ }^{1}$ as shown in Fig. 7(a). Our data show that in the region of bistability $(\sim 1.650 \mathrm{~V})$ the system can be in one of two stable (or metastable) configurations of
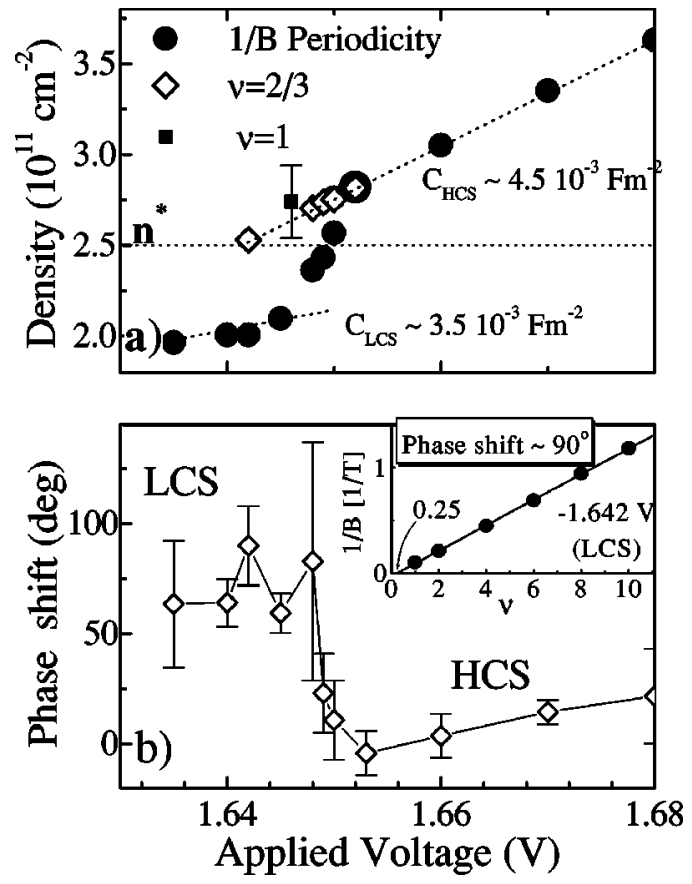

FIG. 7. (a) Density of the $e-h$ layers as a function of the applied voltage. The solid circles are extracted from the $1 / B$ periodicity at $T=1.2 \mathrm{~K}$ (see Fig. 6), whereas the open triangles are obtained from the $\nu=2 / 3$ minima at $T=70 \mathrm{mK}$. The square is obtained from the $\nu=1$ minimum shown in Fig. 3 of Ref. 1. In the critical region, the system can be in one of two states with different density (see also Fig. 3 of Ref. 1). (b) Phase shift of the MO at fixed bias. The inset shows a plot of $1 / B$ vs $\nu$ for $V=1.642 \mathrm{~V}$.

different density, in quantitative agreement with the bistability of the MO upon a sweep of magnetic field (Fig. 3 of Ref. $1)$.

From the values of the density as a function of applied voltage, we can estimate the capacitance per unit area, $e d n / d V$ (where $e$ is the electron charge). As shown in Fig. 2, the capacitance is 4.5 and $3.5 \mathrm{~F} \mathrm{~m}^{-2}$ in the HCS and LCS, respectively, corresponding to a capacitor with interlayer distance of $\sim 25 \mathrm{~nm}$ and $\sim 30 \mathrm{~nm}$, respectively.

We would like to stress that all the differences in the MO clearly show that the LCS and HCS have a qualitatively

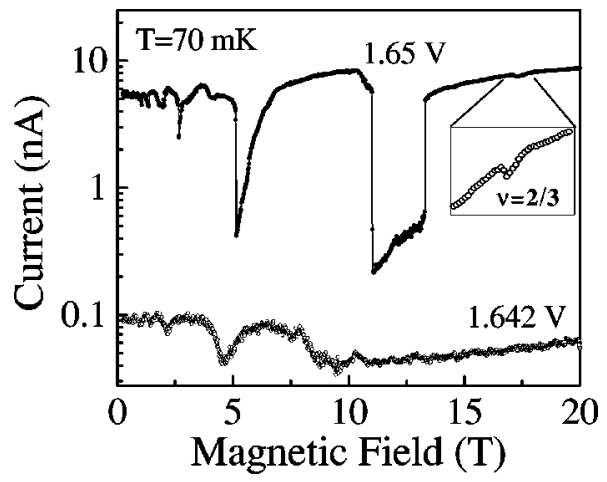

FIG. 8. Magneto-oscillations of the tunnel current at $T$ $=70 \mathrm{mK}$ and fixed bias $V=1.64 \mathrm{~V}$ (LCS) and $1.65 \mathrm{~V}$ (LCS + HCS $)$. The feature at $\nu=2 / 3$ is observed only in the HCS and at $T=70 \mathrm{mK}$. 


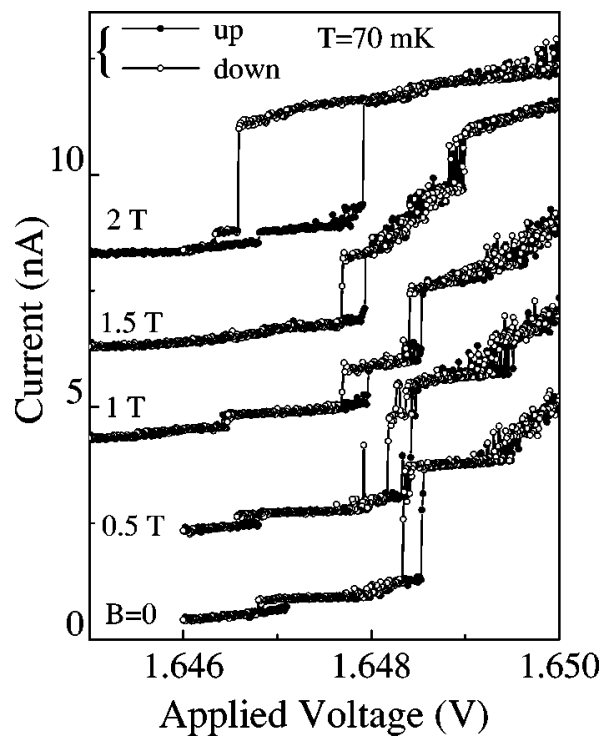

FIG. 9. $I(V)$ curves at $T=70 \mathrm{mK}$ as a function of magnetic field. The curves are taken at steps of $0.5 \mathrm{~T}$ and are offset for comparison. Sweep up and down are shown with solid and open squares, respectively.

different DOS. Already at a first sight in Fig. 6, it is clear that the oscillations of the LCS and HCS are quite distinct in nature. For example, the positions of the maxima differ considerably in the two states, as can be clearly seen for the maxima at $1<\nu<2$ (marked by circles in Fig. 6). Since the maxima in the current occur when the Fermi energy (fixed by the contacts) lies in the middle of a LL (swept by the magnetic field), the observed discontinuity of the maximum positions directly witnesses a qualitative change of the DOS of the LCS and HCS, which is also confirmed by the change of the phase observed from the minimum positions [Fig. 7(b)]. Other qualitative differences include the suppression of the $\nu=1$ minimum and the suppression of the current at high magnetic fields only for the LCS (Fig. 6).

\section{Filling-factor dependence of the bistability}

We report below how the hysteresis in the $I(V)$ curves at $B=0$ (Fig. 1) evolves in the presence of a constant magnetic field perpendicular to the layers. At low magnetic fields (0 $\leq B \leq 2 \mathrm{~T}$ ), the transition from the LCS to the HCS occurs in several steps with complex magnetic field dependence (Fig. 9), and can be resolved only for $T \leqslant 300 \mathrm{mK}$. This behavior is qualitatively similar to the one in the absence of magnetic field shown in Fig. 2(b). The steps could be accurately reproduced, but their detailed size and position depended on the particular mesa measured.

At higher magnetic field, the steps coalesce into a bigger feature and the behavior simplifies considerably. At $B$ $\gtrsim 6 \mathrm{~T}$, both the amplitude and width of the hysteresis increase of one order of magnitude, and they show $1 / B$ periodicity. In particular, a marked suppression occurs around the integer filling factors of the HCS, as can be clearly seen in Fig. 10. In the figure, the curve at $B=11.5 \mathrm{~T}$ (which corresponds to $\nu \approx 1$ ) is offset for comparison.

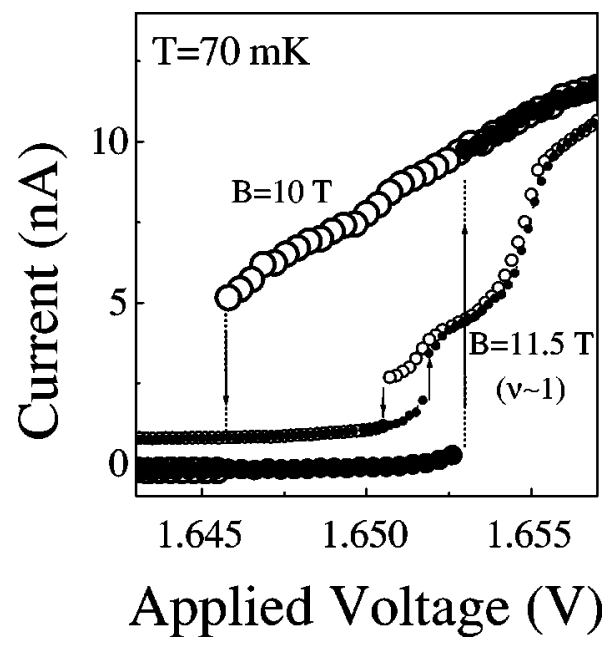

FIG. 10. Bistability of the tunnel current at $B=10 \mathrm{~T}$ and $T$ $=70 \mathrm{mK}$. The solid and open circles refer to sweep up and down, respectively. The smaller circles (offset for comparison) refer to $B$ $=11.5 \mathrm{~T}(\nu \approx 1)$.

We show in Fig. 11 all the $I(V)$ curves measured at $T$ $=1.2 \mathrm{~K}$, at $4.5 \mathrm{~T} \leq B \leq 17 \mathrm{~T}$, with a step of $0.5 \mathrm{~T}$. Sweeps up and down are plotted with solid and dashed lines, respectively. The curves are offset for comparison and clearly oscillate with $1 / B$ periodicity, which is very close to the one of the HCS close to the transition $(1.65 \mathrm{~V})$.

At the integer filling factor, the bistability width and the current discontinuity have minimal size, whereas the voltage position has a maximum. The detailed analysis of all these properties is shown in Fig. 12. In Fig. 12(a), we show the voltage position of the current discontinuity as a function of

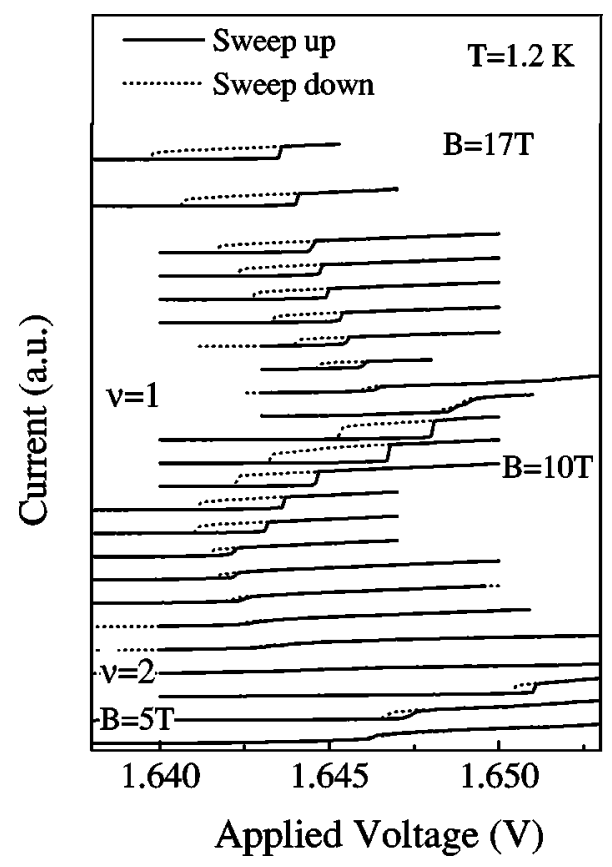

FIG. 11. $I(V)$ curves at $T=1.2 \mathrm{~K}$ as a function of magnetic field. The curves are taken at steps of $0.5 \mathrm{~T}$ and are offset for comparison. Sweep up and down are shown with solid and dotted lines, respectively. 

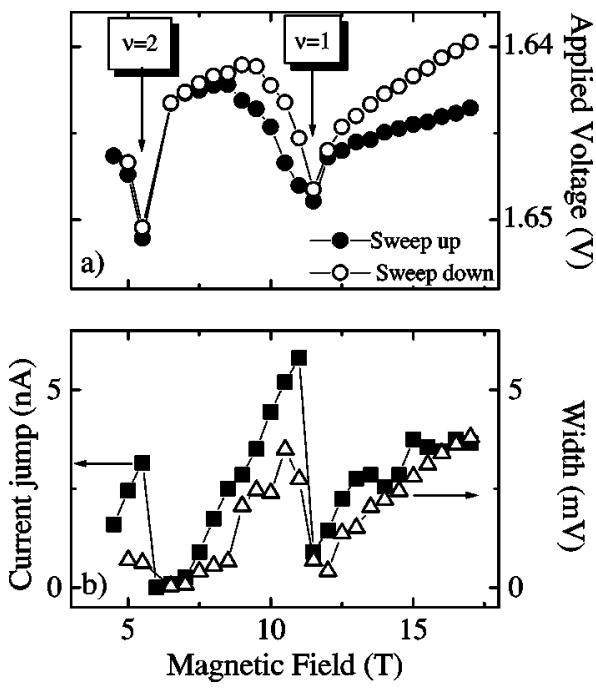

FIG. 12. (a) Voltage position of the current discontinuity. The solid and open circles refer to sweep up and down, respectively. (b) Size of the current discontinuity (squares, left axis) and of the bistability width (open triangles, left axis).

magnetic field. The solid and open circles refer to sweeps up and down, respectively. In Fig. 12(b), we show the current jump (solid squares) and the bistability width (open triangles). All these properties are clearly related to each other, as already seen from the temperature dependence (see Sec. III A).

\section{In-plane magnetic field}

Finally, in order to further investigate the tunneling properties of the HCS and LCS, we have measured the influence of an in-plane magnetic field $B_{\|}$on the $I(V)$ curves, at $T$ $=1.2 \mathrm{~K}$. The measurements are shown in Fig. 13, where we show three representative $I(V)$ curves taken at $B=0,1$, and $3 \mathrm{~T}$ (solid circle, open circle, and open squares, respectively).

The HCS current decreases when $B_{\|}$increases, whereas the LCS current increases for $0.25 \leq B \| \leq 1 \mathrm{~T}$, and decreases $B_{\|} \gtrsim 1$ T. In the inset of Fig. 13, we show the magnetic field behavior of the integrated current in the region $1.65-1.66 \mathrm{~V}$ (HCS) and 1.64-1.65 V (LCS). The suppression of current in the HCS is typical of a $2 \mathrm{DEG},{ }^{29}$ whereas the current increase of the LCS is anomalous. As a result of this difference between the LCS and HCS, the feature in the $I(V)$ curves at $B=0$ is clearly smeared by in-plane fields $B \geq 0.25 \mathrm{~T}$, as shown in Fig. 13.

\section{DISCUSSION}

\section{A. Indirect-exciton picture}

All the measurements reported above strongly suggest that the observed discontinuity and bistability both arise from a transition between the LCS and HCS, which is strongly dependent on the screening properties of the 2D layers. As discussed in the Introduction, at low densities $\left(d_{e-e} \gg d_{e-h}\right)$, the system is a diluted gas of spatially indirect

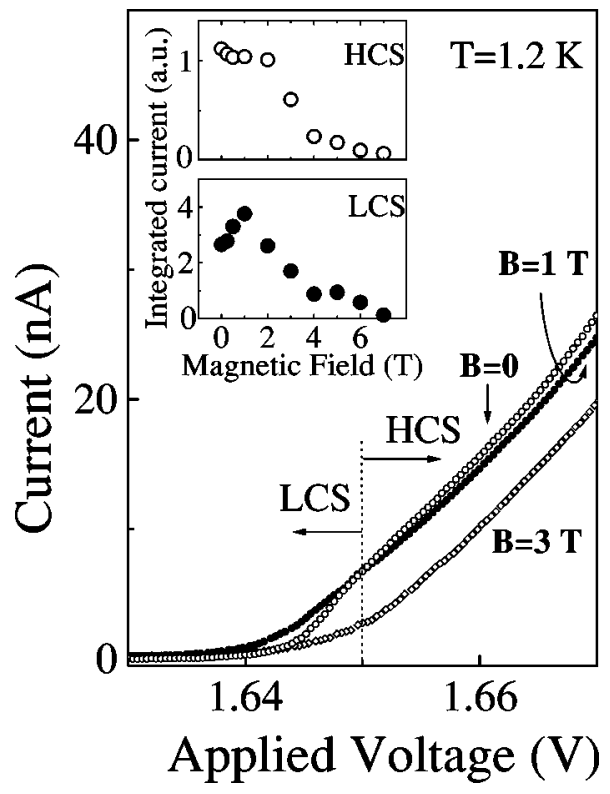

FIG. 13. $I(V)$ curves at $T=1.2 \mathrm{~K}$ as a function of magnetic field parallel to the layers. Solid circles, open circles, and open squares refer to $B=0,1 \mathrm{~T}$, and $3 \mathrm{~T}$, respectively. In the inset, the integrated current in the regions $1.65-1.66 \mathrm{~V}$ (HCS) and 1.64-1.65 $\mathrm{V}$ (LCS) is shown.

excitons, ${ }^{20}$ whereas at high densities $\left(d_{e-e} \leqslant d_{e-h}\right)$ the $e$ and $h$ layers uncouple into separate 2DEG and 2DHG (twodimensional hole gas). ${ }^{23}$

We identify the HCS with the uncoupled $e$ and $h$ gases, since it behaves regularly in the presence of perpendicular ${ }^{23}$ and in-plane ${ }^{29}$ magnetic fields. In contrast, the LCS behaves peculiarly and has a different DOS, as discussed in Sec. III B. As we discuss below, we believe that the LCS can be described as indirect excitons.

\section{Current magneto-oscillations}

Our assignment for the LCS is supported by the behavior of the MO shown in Sec. III B, in particular by the absence of fractional features and by the phase shift of the MO. Indeed, the indirect excitons (IE's) are not expected to form the incompressible Laughlin fluids, since they are bound by the attractive $e-h$ attraction. In contrast, the uncoupled $e-h$ layers minimize $e-e(h-h)$ interactions by forming composite fermions at the fractional filling factor, ${ }^{30}$ as we observe in the HCS.

The phase shift in the MO confirms this picture and directly witnesses a change in the DOS, which also results in the general behavior of the MO (Figs. 6 and 7). A comprehensive study of a gas of indirect excitons in high magnetic fields has been recently reported by Lozovik and Ruvinsky. ${ }^{31}$ The main consequences of the $e-h$ Coulomb interaction on free-particle LL's are a shift to lower energies (exciton binding) and the appearance of in-plane dispersion originating from the coupling between center of mass and relative motion. This property accounts very well for the fact that we observe a qualitative difference in the DOS of the LCS and HCS (Fig. 6). 
In addition, a similar phase change has been observed by Volkov et al. $^{32}$ in the magneto-oscillations of the luminescence intensity of direct excitons, and it was explained by the presence of exciton states below the free-particle LL's. The phase shift disappeared at high excitation, as the excitons were destroyed by screening. Although providing a consistent picture, the explanation given in Ref. 32 is based on energy levels rather than on level degeneracy. ${ }^{33}$ Our experimental results are consistent with Ref. 32 and are based on the assumption that the electrons, although forming excitons, obey a Fermi statistics. This surprising behavior, which is a direct consequence of the interpretation of the current magneto-oscillations in terms of the carrier density, is also in agreement with optical studies of the IE linewidth at low density. ${ }^{15}$

\section{2. $e-h$ distance}

The data of the current (Fig. 2) and the capacitance (Fig. 7) are consistent with an IE $e-h$ distance of abound $30 \mathrm{~nm}$, which is more than $10 \%$ higher that in the HCS. We performed self-consistent calculations in the limit of low densities, but we could not describe such behavior. The reason for this discrepancy may be that the IE's are actually strongly interacting, or may lay in some subtleties in the selfconsistent rearrangement of the band profile. It might also happen that, in the LCS, thermodynamic quasiequilibrium is not reached, and part of the potential is dropping in the doping regions.

\section{Current discontinuity}

Within the same simple model, we can explain the low current of the LCS at $B=0$. The total current flowing through the device is proportional to both the density and tunneling probability of the electrons. Both these factors are smaller in the LCS than in the HCS.

The smaller density $(\sim 10 \%)$ was deduced experimentally and we discussed extensively in Sec. III B (Figs. 7 and 3 of Ref. 1). The electron tunneling probability is proportional to the in-plane overlap between the wave functions of initial (electron in an unbound or exciton state) and final states (plane-wave electrons in the collector). ${ }^{34,35}$ In our case, the wave functions of the initial and final states are not known, but since we find that the $e-h$ distance is larger in the LCS than in the HCS (see the previous section), we expect the LCS tunneling probability to be smaller than that of the HCS.

We observed that the LCS current increases in the presence of moderate parallel magnetic fields $B_{\|}$. Although such an increase does not violate the preceding interpretation, we are not able to quantify the effect of $B_{\|}$(which might substantially alter the electronic configuration ${ }^{36}$ ) on the tunneling of indirect excitons. It is, anyway, notable that this behavior is remarkably in contrast with the tunneling of free carriers, such as in the HCS. In the latter case, the tunnel current always decreases as a consequence of the squeezing of the electron wave function in the growth direction and because of the mismatch in $k$ space between the initial and final states. ${ }^{29}$

\section{Exciton binding energy}

In our interpretation, the LCS is characterized by a binding energy $E_{b}$, which we estimate from the temperature $T^{*}$ that suppresses both the bistability and the discontinuity. For $B=0$ and $B=10 \mathrm{~T}, T^{*}$ was shown to be $\sim 300 \mathrm{mK}$ and $\sim 10 \mathrm{~K}$, respectively, and roughly proportional to the bistability width (which saturates for $T \$ 300 \mathrm{mK}$ ). We can therefore estimate the ratio $E_{b}\left(\begin{array}{ll}10 & \mathrm{~T}\end{array}\right) / E_{b}\left(\begin{array}{ll}0 & \mathrm{~T}\end{array}\right) \sim 20$.

It is very difficult to estimate the absolute value of $E_{b}$ since the proportionality factors are not known a priori. It is remarkable that the temperature dependence, in particular $T^{*}$, suggests that the bistability width is directly related, via a leverage factor, to the binding energy. With a leverage factor of 1, we obtain an estimation of an upper limit: $E_{b}$ $\sim 0.3 \mathrm{meV}$ at $B=0$ and $E_{B} \sim 5 \mathrm{meV}$ at $B \sim 10 \mathrm{~T}$; if we assume that $E_{b} \sim K T^{*}$ ( $K$ is the Boltzmann constant), we find a lower limit: $E_{b} \sim 0.03 \mathrm{meV}$ at $B=0$ and $E_{B}$ $\sim 0.5 \mathrm{meV}$ at $B \sim 10 \mathrm{~T}$. Both these values, however, should be considered only as approximate, though they are in reasonable agreement with the existing literature. The value deduced at $B=0$ agrees, for example, with Ref. 37 (screening additionally decreases the IE binding energy), whereas the increase of $E_{b}$ with magnetic field qualitatively agrees with the $\operatorname{expression}^{38} \quad E_{b}(B) \sim 1 / \sqrt{d^{2}+l_{B}^{2}}$, where $\quad l_{B}$ $=25.66 \mathrm{~nm} / \sqrt{B}$ is the magnetic length.

We stress that the analysis reported in this section regards only the general increase in size of the bistability in a magnetic field. We believe that the filling-factor dependence of the hysteresis has a different explanation, which will be described in the following section.

\section{Description of the hysteresis}

When the bias is increased in the LCS, the $e-e(h-h)$ separation decreases until $d_{e-e} \sim d_{e-h} \approx 25 \mathrm{~nm}$; at this point, the wave function overlap activates screening, and the indirect excitons dissociate. This transition is abrupt and occurs with a self-consistent rearrangement of the band profile, which induces an higher density; since we are not able to calculate the wave functions of the IE's, however, we cannot give any estimation of the density discontinuity.

In contrast, when the bias is decreased in the HCS, $d_{e-e}$ increases until the exciton gas forms. The latter transition occurs at a lower bias than the former one because screening suppresses the formation of indirect excitons. In other words, the system has a memory that arises from the interplay between screening and exciton binding, which gives rise to the hysteresis.

Indeed, we have shown in Fig. 11 that both the bistability and the discontinuity are strongly suppressed at the integer filling factor, when the screening is minimal ${ }^{30}$ and the carriers are localized efficiently by the magnetic field also in the HCS. At integer filling factors, the HCS current therefore decreases, and the transition to the LCS becomes smoother and occurs at higher biases. The magnetic field affects much less the screening properties of the LCS, since those states are already localized by the $e-h$ attraction.

The presence of two or more steps in the current at low magnetic fields could arise from the interaction among exci- 
tons: the transition would occur via intermediate states that cannot be described by our simple model. Another possibility is that the different features arise from different areas of the sample, because of inhomogeneities of the device.

\section{Thermodynamical phase transition}

Our measurements of the density discontinuity (Fig. 7) suggest that the transition is thermodynamical. In particular, since in our structure the chemical potential $\mu_{e-h}(n)$ is equal to $e V$, where $V$ is the applied voltage (Fig. 1), our data show that at the bistability, the HCS and LCS have different densities at the same chemical potential. Such a nonlinearity in $\mu_{e-h}(n)$ would be accompanied in our structure by a selfconsistent rearrangement of the band profile. In other words, the hysteresis could be described as a first-order phase transition. ${ }^{2}$

In addition, we notice that the magnetic field behavior of the width of the bistability (Fig. 12) bears a strong resemblance to the behavior of the critical temperature for the excitonic state predicted in Refs. 9 and 17, with a critical temperature that is minimal at the integer filling factor. An interpretation of our data in terms of collective properties of the indirect excitons, however, is beyond the purposes of this paper.

\section{B. Possibility of charge localization}

The energy scales of the hysteresis, which can be estimated from the temperature dependence, are also typical of shallow binding; in this respect, localization of holes ${ }^{39}$ due to interface roughness could occur in our device. We cannot exclude the possibility that some of the excitons are localized by interface defects, but this would not modify essentially the description. However, we believe that localization alone (whether of excitons or free carriers) can only explain with difficulty the particular conditions at which the feature is observed $\left(d_{e-e} \sim d_{e-h}\right)$, the mechanism of bistability, the filling factor dependence of the hysteresis, and the difference of the HCS and LCS DOS (Fig. 6).

In addition, as discussed in Sec. II, the results shown here could be only obtained in samples designed in order to optimize the hole in-plane mobility (which we could not directly measure). Such a sample might have surface corrugation along the $[\overline{2} 33]$ direction, ${ }^{40}$ which could affect the hole transport properties. It is unlikely, however, that such corrugation could affect the localization properties of the holes, since its typical scale of $3 \mathrm{~nm}$ is much smaller than the other lengths involved in our experiment.

\section{Discussion of known mechanisms for bistability}

As we discuss below, our experimental observations cannot be explained by previously reported mechanisms of bistability on similar structures.

Impurity-related trapping-detrapping processes are known to lead to bistability in bulk GaAs; ${ }^{41}$ in our case, however, the observed dependence on the filling factor and the suppression by a modest in-plane magnetic field unambiguously show a $2 \mathrm{D}$ origin.
Nor can the bistability be ascribed to trapping-detrapping mechanisms involving 2D states, as could arise from resonant tunneling via $X$-valley quantum well states ${ }^{42}$ or impurity states in the AlAs layer. We do observe the former as much weaker and broader features in the $I(V)$ at higher bias ( $V$ $\approx 1.7 \mathrm{~V}$ in Fig. 2, in agreement with Ref. 22). Impurity states in the barrier have been studied in $12 \mathrm{~nm}$ GaAs/AlAs/ GaAs $n-i-n$ single-barrier structures with intentional Si doping in the AlAs layer, ${ }^{27,43,44}$ the $I(V)$ curves of these samples show broad tunneling features that evolve into peaks in the presence of a perpendicular magnetic field and shift to lower voltages for $B \gtrsim 10 \mathrm{~T}$, whereas the feature that we observe is bistable, discontinuous, and does not shift globally in voltage for magnetic fields up to $17 \mathrm{~T}$.

Our experimental data are qualitatively very similar to the single-barrier heterostructure hot-electron diode ${ }^{45}$ in which an S-shaped nonlinearity arises because of the competition between two transport regimes: tunneling through the barrier in the low-conductivity state and thermionic emission in the high-conductivity states. Despite the difference with our device, in which thermionic emission is totally negligible, we believe that the general mechanism for the bistability is very similar and arises from the competition between the LCS and HCS.

\section{SUMMARY}

In conclusion, we have reported on a discontinuous and bistable feature in the tunnel current of strongly interacting 2D $e$ and $h$ layers. Both the discontinuity and the distability width are enhanced by magnetic fields $B \sim 10 \mathrm{~T}$ perpendicular to the layers and suppressed by in-plane magnetic fields $B \sim 1 \mathrm{~T}$. They correspond to a discontinuity in the $e-h$ density and in the phase of the current magneto-oscillations. Whereas the HCS has the expected properties of the uncoupled $e-h$ layers, the LCS behaves anomalously under all circumstances.

We identify the HCS with the uncoupled $e-h$ layers and the LCS with a gas of spatially indirect excitons with binding energy $0.03 \mathrm{meV} \lesssim E_{b} \lesssim 0.3 \mathrm{meV}$ and $0.5 \mathrm{meV} \lesssim E_{b}$ $\$ 5 \mathrm{meV}$ at $B=0$ and $B=10 \mathrm{~T}$, respectively.

We interpret the bistability as a transition between the two regimes, which arises because of the competition between in-plane screening and interlayer $e-h$ attraction. The hysterisis is considerably affected by the screening properties of the 2D layers in a magnetic field and therefore strongly depends on the HCS filling factor.

\section{ACKNOWLEDGMENTS}

This work was part of a research program of the "Stichting voor Fundamenteel Onderzoek der Materie" (FOM) financially supported by NWO (The Netherlands). It has also been supported by the European Commission under Contract No. ERBFMGECT950079. We are grateful to M. Fromhold and F. Sheard for useful discussions. 
${ }^{1}$ A. Parlangeli et al., Phys. Rev. B 60, 13302 (1999); Prog. Solid State Chem. 164, 587 (1997).

${ }^{2}$ A. Parlangeli et al., in Workbook of the 13th International Conference on the Electronic Properties of Two-Dimensional Systems, Ottawa, 1999 [Physica E (Amsterdam) 6, 832 (2000)].

${ }^{3}$ A. Parlangeli et al., Physica B 256-258, 531 (1998).

${ }^{4}$ L. V. Butov, A. Zrenner, G. Abstreiter, G. Böhm, and G. Weimann, Phys. Rev. Lett. 73, 304 (1994).

${ }^{5}$ L. V. Butov and A. I. Filin, Phys. Rev. B 58, 1980 (1998).

${ }^{6}$ L. V. Butov, A. A. Shashkin, V. T. Dolgopolov, K. L. Campman, and A. C. Gossard, Phys. Rev. B 60, 8753 (1999).

${ }^{7}$ Yu. E. Lozovik and O. L. Berman, Zh. Éksp. Teor. Fiz. 84, 1879 (1999) [JETP 84, 1027 (1997)]; Yu. E. Lozovik, O. L. Berman, and V. G. Tsvetus, Pis'ma Zh. Éksp. Teor. Fiz. 66, 332 (1997) [JETP Lett. 66, 355 (1997)].

${ }^{8}$ I. V. Lerner and Yu. E. Lozovik, Pis'ma Zh. Éksp. Teor. Fiz. 27, 497 (1978) [JETP Lett. 27, 467 (1978)].

${ }^{9}$ I. V. Lerner and Yu. E. Lozovik, J. Low Temp. Phys. 38, 333 (1980).

${ }^{10}$ S. I. Shevchenko, Fiz. Nizk. Temp. 2, 505 (1976) [Sov. J. Low Temp. Phys. 2, 251 (1976)].

${ }^{11}$ X. M. Chen and J. J. Quinn, Phys. Rev. Lett. 67, 895 (1991).

${ }^{12}$ D. Yoshioka and A. H. MacDonald, J. Phys. Soc. Jpn. 59, 4211 (1990).

${ }^{13}$ D. Yoshioka and H. Fukuyama, J. Phys. Soc. Jpn. 45, 137 (1978).

${ }^{14}$ T. Fukuzawa, E. E. Mendez, and J. M. Hong, Phys. Rev. Lett. 66, 3066 (1990).

${ }^{15}$ J. A. Kash et al., Phys. Rev. Lett. 66, 2247 (1991).

${ }^{16}$ J. E. Golub, K. Kash, J. P. Harbison, and L. T. Florez, Phys. Rev. B 45, 9477 (1992).

${ }^{17}$ Y. Kuramoto and C. Horie, Solid State Commun. 25, 713 (1978).

${ }^{18}$ U. Sivan, P. M. Solomon, and H. Shtrikman, Phys. Rev. Lett. 68, 1196 (1992).

${ }^{19}$ B. E. Kane, J. P. Eisenstein, W. Wegscheider, L. N. Pfeiffer, and K. W. West, Appl. Phys. Lett. 65, 3266 (1994).

${ }^{20}$ B. R. A. Neves et al., in Proceedings of the $23 \mathrm{rd}$ International Conference on Physics of Semiconductors, Berlin, 1996, edited by M. Scheffler and R. Zimmermann (World Scientific, Singapore, 1996), p. 2275.

${ }^{21}$ M. Anderson, J. R. Fisher, M. R. Matthews, C. E. Wieman, and E. A. Cornell, Science 269, 198 (1995).

${ }^{22}$ J. J. Finley et al., Superlattices Microstruct. 23, 513 (1998).

${ }^{23}$ R. Teisser et al., Phys. Rev. B 51, 5562 (1995).

${ }^{24}$ T. W. Hickmott, Phys. Rev. B 32, 6531 (1985).
${ }^{25}$ K. S. Chan, F. W. Sheard, G. A. Toombs, and L. Eaves, Phys. Rev. B 56, 1447 (1997).

${ }^{26}$ E. Böckenhoff, K. v. Klitzing, and K. Ploog, Phys. Rev. B 38, R10 120 (1988).

${ }^{27}$ R. K. Hayden et al., J. Phys. Soc. Jpn. 66, 2228 (1997).

${ }^{28}$ The determination of the density, and therefore $d_{e-h}$, will be discussed in Sec. III B.

${ }^{29}$ T. M. Fromhold, F. W. Sheard, and G. A. Toombs, Surf. Sci. 228, 437 (1990).

${ }^{30}$ T. Chakraborty and P. Pietilainen, The Quantum Hall Effects, 2nd ed. (Springer-Verlag, Berlin, 1995).

${ }^{31}$ Yu. E. Lozovik and A. M. Ruvinsky, Phys. Lett. A 227, 271 (1997).

${ }^{32}$ O. V. Volkov, V. E. Zhitomirskii, I. V. Kukushkin, K. von Klitzing, and K. Eberl, Pis'ma Zh. Éksp. Teor. Fiz. 64, 719 (1996) [JETP Lett. 64, 774 (1996)].

${ }^{33}$ An appropriate discussion of the MO must take into account the degeneracy of the energy levels. A typical example is a $2 \mathrm{D}$ hole gas in a magnetic field. Such a system has a very complicated energy structure, but shows a very precise $1 / B$ periodicity.

${ }^{34}$ J. Bardeen, Phys. Rev. Lett. 6, 57 (1961).

${ }^{35}$ S. M. Cao and M. Willander, J. Appl. Phys. 81, 6221 (1997).

${ }^{36}$ S. Q. Murphy, J. P. Eisenstein, G. S. Boebinger, L. N. Pfeiffer, and K. W. West, Phys. Rev. Lett. 72, 728 (1994).

${ }^{37}$ Yu. E. Lozovik and V. N. Nishanov, Fiz. Tverd. Tela (Leningrad) 18, 3267 (1976) [Sov. Phys. Solid State 18, 1905 (1976)].

${ }^{38}$ A. B. Dzyubenko and A. L. Yablonskii, Phys. Rev. B 53, 1 (1996).

${ }^{39}$ The possibility of electron localization is ruled out by the fact that the feature is absent in $n-i-n$ samples. The tunneling properties of holes are much less known. Anyway, electrons are less sensitive to localization than holes, because of their smaller effective mass.

${ }^{40}$ M. Wassermeier, J. Sudijono, M. D. Johnson, K. T. Leung, and B. G. Orr, Phys. Rev. B 51, 14721 (1995).

${ }^{41}$ M. A. Lampert and P. Mark, Current Injection in Solids (Academic Press, New York, 1970).

${ }^{42}$ R. Beresford, L. F. Luo, W. I. Wang, and E. E. Mendez, Appl. Phys. Lett. 55, 1555 (1989).

${ }^{43}$ H. Fukuyama, T. Waho, and M. Yamamoto, Jpn. J. Appl. Phys., Part 1 36, 4267 (1997).

${ }^{44}$ I. E. Itskevich, L. Eaves, P. C. Main, M. Henini, and G. Hill, Phys. Rev. B 57, 7214 (1998).

${ }^{45}$ R. Stasch, R. Hey, M. Asche, A. Wacker, and E. Scöll, J. Appl. Phys. 80, 3376 (1996). 